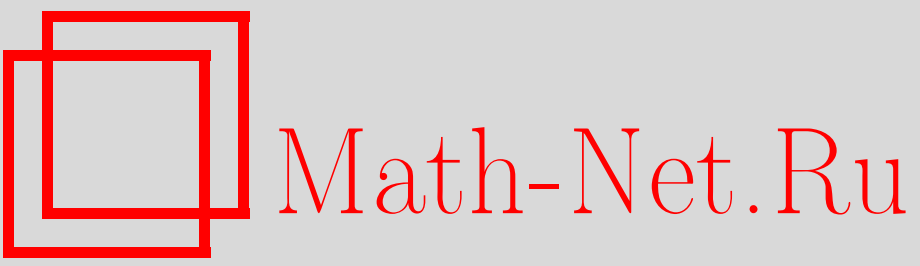

В. В. Горюнов, Унитарные группы отражений, связанные с особенностями функций с циклической симметрией, УМН, 1999, том 54, выпуск 5, 3-24

DOI: https://doi.org/10.4213/rm202

Использование Общероссийского математического портала Math-Net.Ru подразумевает, что вы прочитали и согласны с пользовательским соглашением

http://www.mathnet.ru/rus/agreement

Параметры загрузки:

IP: 3.85 .5 .30

26 апреля 2023 г., 17:01:14 


\title{
УНИТАРНЫЕ ГРУППЫ ОТРАЖЕНИЙ, СВЯЗАННЫЕ С ОСОБЕННОСТЯМИ ФУНКЦИЙ С ЦИКЛИЧЕСКОЙ СИММЕТРИЕЙ
}

\author{
В. В. ГорЮнов
}

Появление конечных групп, порожденных евклидовыми отражениями, в самых различных задачах теории особенностей стало традицией с тех пор, как В.И. Арнольд обнаружил их связь с классификацией критических точек функций [1], [2]. Мы показываем, что ряд конечных групп, порожденных унитарными отражениями, столь же естественно связан с особенностями функций, на этот раз с функциями, инвариантными относительно унитарного отражения конечного порядка. Чтобы продемонстрировать это, мы рассматриваем ростки функций на многообразии с краем и поднимаем их на циклическое накрытие данного многообразия, разветвленное над краем. Наша конструкция приводит к новому определению корней рассматриваемых групп, а также к косо-эрмитовым аналогам этих групп.

Библиография: 22 названия.

\section{СОДЕРЖАНИЕ}

$\S 1$. Изолированные особенности функций

5

1.1. Милноровский слой ростка функции

1.2. Исчезающие циклы

1.3. Группа монодромии особенности

1.4. Классифитания простых особенност

$\S 2$. Краевые особенности

2.1. Исчезающие циклы и полуциклы

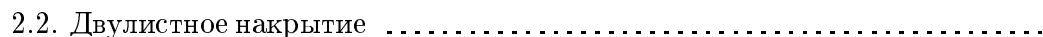

2.3. Простые краевые особенности

$\S 3$. Конечные группы, порожденные унитарными отражениями

$\S 4$. Циклическое накрытие функции на многообразии с краем

$\S 5$. Функции с циклической симметрией

5.1. Эллиптические особенности ... . . . . . . . . . . . . . . . . . . . . . . .

5.2. Диаграммы пересечений

5.3. Окончание доказательства теоремы 5.2

5.4. Особенность $B_{2}^{(3,3)}$

5.5. Целочисленность

5.6. Стягивание диаграмм

Список литературы

С) В.В. Горюнов 
Более двадцати лет назад В. И. Арнольд поставил вопрос о реализациях конечных групп, порожденных унитарными отражениями, в теории особенностей. Настоящая работа является первым шагом на пути решения этой проблемы.

В 1972 году Арнольд обнаружил естественное взаимно-однозначное соответствие между простыми особенностями функций и группами Вейля $A_{k}, D_{k}$ и $E_{k}$, диаграммы Дынкина которых не имеют кратных ребер [1]. Было установлено, что в гомологиях милноровского слоя простой функции имеется отмеченный базис, граф пересечений которого - как раз соответствуюшая диаграмма Дынкина. Группа монодромии простой особенности - в точности сопоставляемая ей группа Вейля. Множество нерегулярных орбит комплексификации групшы Вейля изоморфно дискриминанту отвечающей ей функции.

В 1978 году Арнольд распространил свои результаты на соответствие между функциями на многообразии с краем и группами $B_{k}, C_{k}$ и $F_{4}$, в диаграммах Дынкина которых встречаются двойные ребра [2]. Основной идеей его конструкции был переход от относительных гомологий пары слоев Милнора функции и ее ограничения на край к пространству с корректно определенной формой пересечений. С этой целью Арнольд ввел в рассмотрение двулистное накрытие многообразия, разветвленное над краем.

Последняя группа Вейля, $G_{2}$, проявилась в теории особенностей как функция с симметрией $S_{3}[2]$.

За открытиями Арнольда последовал увенчавшпийся успехом поиск реализаций в теории особенностей групп отражений $H_{3}, H_{4}$ и $I_{2}(p)$ [14], [18], [13].

При анализе подхода Арнольда к определению формы пересечений краевой особенности возникает естественньй вопрос: что дает замена двулистного накрытия произвольным циклическим? Ответ на этот вопрос приводит к особенностям, связанным с рядом конечных групп, порожденных унитарными отражениями. Обнаруживающаяся связь обобщает открытую Арнольдом связь между функциями и группами Вейля.

Реализующиеся у нас групшы почти полностью выделяются из классификационного списка Шепарда-Тодда [19] следуюшими двумя свойствами:

- группы порождены $n$ отражениями в $\mathbb{C}^{n}$,

- степени базисных инвариантов симметричны относительно их среднего арифметического.

"Почти" означает, что мы опускаем $H_{3}, H_{4}$ и некоторые двумерные группы.

Было бы очень интересно понять, какие внутренние свойства выделяют появляющиеся в нашей ситуации группы отражений из всего списка групп Шепарда-Тодда.

Следует отметить, что пять из рассматриваемых нами унитарных групा отражений уже появлялись в работе Гивенталя [12], но остались там неопознанными. Это, в наших обозначениях, групшы $A_{k}^{(m)}$. Они отвечают специальным значениям параметра в представлении Бюрау групп кос. Представления Бюрау рассматривались в [12] в контексте, весьма близком к нашему. Некоторые элементы нашей конструкции появлялись также в [10].

Заметим также, что классификация циклически симметричных функций - далеко не новый вопрос в теории особенностей. Он, к примеру, рассматривался Вассерманом [22] и Тибаром [21]. В частности, работа [21] содержит особенности, которые мы обозначаем $B_{k}^{(m)}$. Несмотря на это, связь с унитарными группами отражений замечена не была. 
Остановимся коротко на содержании настоящей статьи.

В первом и втором параграфах мы напоминаем основные факты о простых функциях на гладком многообразии и на многообразии с краем, делая акцент на их связь с группами Вейля.

В третьем параграфе мы останавливаемся на тех из унитарных групп отражений, которые появятся у нас впоследствии в связи с особенностями.

В $\S 4$ рассматриваются конечные циклические накрытия многообразия с краем с ветвлением над краем. Поднимая функцию на пространство накрытия, мы определяем отмеченньй базис в гомологиях циклически симметричного милноровского слоя поднятия, которьй согласуется с действием группы накрытия.

Параграф 5 посвящен функциям на комплексном линейном пространстве, инвариантным относительно действия конечной циклической группы $\mathbb{Z}_{m}$, сохраняющего гиперплоскость. Мы классифицируем эллиптические симметричные функции, т.е. функции с конечной группой монодромии. Такая классификация оказывается здесь более естественной, чем традищионная классификация простых особенностей. Наши унитарные групшы отражений реализуются как группы монодромии, действуюшие на подпространствах, отвечающих характерам групп $\mathbb{Z}_{m}$ в гомологиях симметричных слоев Милнора эллиптических особенностей.

В п. 5.5 отмечается, что геометрический базис подпространства, отвечающего характеру, обладает свойством целочисленности, весьма сходным с тем, что есть у системы корней группы Вейля. Похоже, что это свойство осталось незамеченным в теории групп, где в интересующих нас случаях главной целью обычно было выбрать ортобазис, в котором матрища эрмитовых произведений была бы вешественной (ср. $[7],[9])$. При нашем подходе эта матрица не является вешественной (ср. [17]) и корни естественным образом различаются по длине.

Всякая циклически инвариантная эллиптическая функция проста как несимметричная особенность. Это вписьвает соответствующую ей унитарную группу в некоторую группу Вейля. В п. 5.6 мы приводим интерпретацию этого факта на языке диаграмм Дынкина. Описываемая нами конструкция обобщает операцию складывания диаграмм, с помошью которой, например, из канонической диаграммы $A_{2 k-1}$ получается каноническая диаграмма $B_{k}$.

Я благодарен Алуну Моррису, Гюнтеру Малле и Терри Уоллу за помощь с литературой по унитарньм группам отражений.

\section{§1. Изолированные особенности функций}

Начнем с необходимых сведений из теории особенностей.

1.1. Милноровский слой ростка функции. Рассмотрим росток голоморфной функции $f:\left(\mathbb{C}^{n+1}, 0\right) \rightarrow(\mathbb{C}, 0)$ с изолированной критической точкой в нуле (мы называем точку критической, если дифференциал функции в ней равен нулю). В дальнейшем мы будем отождествлять росток $f$ с его представителем.

Рассмотрим достаточно малый замкнутьй шар $B \subset \mathbb{C}^{n+1}$ с центром в точке 0 . Выберем ненулевое комплексное число $\varepsilon$, модуль которого достаточно мал по сравнению с радиусом шара $B$. 
ОПРЕДЕЛЕНИЕ 1.1. Слоем Милнора ростка $f$ назьвается многообразие $V=$ $\{f=\varepsilon\} \cap B$.

Милноровский слой является гладким $2 n$-мерным многообразием с краем. Напомним классический результат Милнора [15]:

Tеорема 1.2. Слой Милнора изолированной особенности функции $f$ гомотопически әквивалентен букету конечного числа $n$-мерных сфер.

ОПРЕДЕЛЕНИЕ 1.3. Число $\mu=\mu(f)$ этих сфер назьвается числом Милнора функции $f$.

1.2. Исчезающие циклы. Базис $n$-мерной группы приведенных гомологий $\bar{H}_{n}(V, \mathbb{Z})$ слоя Милнора может быть получен следующим образом.

Напомним, что критическая точка $p$ функции $g$ назьвается морсовской, если матрица вторых производных функции $g$ в точке $p$ невырождена. В окрестности такой точки можно выбрать координаты $x_{0}, \ldots, x_{n}$ с центром в $p$ так, что

$$
g=x_{0}^{2}+\cdots+x_{n}^{2}+g(p) .
$$

Рассмотрим морсификацию $\widetilde{f}$ функции $f$, т.е. малое возмущение функции $f$, имеющее только морсовские критические точки с различными критическими значениями. Мы предполагаем, что функция $\widetilde{f}$ достаточно близка к $f$, что, в частности, гарантирует, что число критических точек функции $\widetilde{f}$ равно $\mu$ и что все они лежат в $B$.

Возьмем регулярное значение $* \in \mathbb{C}$ функции $\widetilde{f}$. Уровень $\{\widetilde{f}=*\} \cap B$ диффеоморфен слою Милнора функции $f$. Обозначим его той же буквой $V$.

Рассмотрим гомотопию многообразия $V$, определенную вдоль несамопересекающегося пути $\gamma$ в $\mathbb{C}$, который соединяет точку $*$ с критическим значением $c$ функции $\widetilde{f}$ и не проходит через остальные критические значения (рис. 1). При приближении к $c$ мы получаем то же локальное изменение некритического уровня функции $\tilde{f}$, что происходит с многообразием $x_{0}^{2}+\cdots+x_{n}^{2}=\delta$ в $\mathbb{C}^{n+1}$, когда положительное число $\delta$ стремится к нулю. В последнем случае $n$-мерная сфера, заданная указанным уравнением в вещественном пространстве $\mathbb{R}^{n+1}=\left\{\operatorname{Im} x_{i}=0\right\}$, стягивается в точку. Это дает нам $n$-мерную сферу в $V$, называемую исчезающим ииклом.

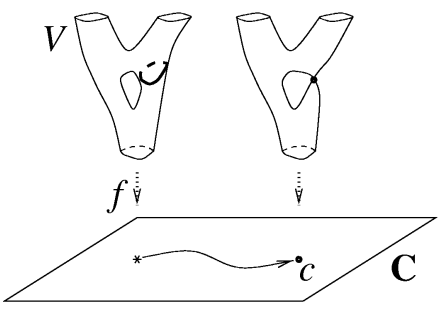

Р ис. 1. Исчезающий в критической точке цикл

Соединим теперь точку $*$ в $\mathbb{C}$ со всеми $\mu$ критическими значениями функции $\tilde{f}$ звездной системой из $\mu$ путей, аналогичных описанному выше. Набор из $\mu$ исчезающих циклов в $V$, определенньй этой системой путей, называется отмеченным набором исчезающих циклов. 
ТеОРема 1.4 [4]. Отмеченный набор исчезающих ииклов образует базис в $\bar{H}_{n}(V, \mathbb{Z})$.

ПримеР 1.5. Рассмотрим функцию $f=z^{3}$ на комплексной прямой. Возьмем в качестве ее морсификации функцию $\widetilde{f}=z^{3}-3 z$ и нулевой уровень функции $\widetilde{f}$ в качестве слоя Милнора $V$. Тогда $\bar{H}_{0}(V)=\left\{\alpha_{1}[-\sqrt{3}]+\alpha_{2}[0]+\alpha_{3}[\sqrt{3}], \sum \alpha_{i}=0\right\} \simeq \mathbb{Z}^{2}$. Критические значения морсификации есть 2 и -2 . В качестве системы путей из точки * = 0 в критические значения возьмем два прямолинейных отрезка. Для такой системы исчезаюшими циклами будут $[0]-[-\sqrt{3}]$ и $[\sqrt{3}]-[0]$. Матрица пересечений этого отмеченного набора исчезаюших циклов есть матрица Картана $A_{2}$.

В общем случае индекс самопересечения исчезающего цикла е равен

$$
\langle e, e\rangle=\left\{\begin{aligned}
0, & n \text { нечетно, } \\
2, & n \equiv 0(\bmod 4), \\
-2, & n \equiv 2(\bmod 4) .
\end{aligned}\right.
$$

1.3. Группа монодромии особенности. Петля в $\mathbb{C}$ c началом в точке $*$, не проходящая через критические значения $c_{1}, \ldots, c_{\mu}$ функции $\widetilde{f}$, определяет автоморфизм слоя Милнора $V$ и, следовательно, автоморфизм групшы $\bar{H}_{n}(V)$. Образ представления на $\bar{H}_{n}(V)$ фундаментальной группы $\pi_{1}\left(\mathbb{C} \backslash\left\{c_{i}\right\}_{i=1}^{\mu}, *\right)$ называется группой монодромии изолированной особенности функции $f$. Он не зависит от всевозможных произволов в построении.

Звездная система путей из предыдущего пункта определяет систему образуюших группы монодромии следуюшим образом. Несамопересекаюшийся путь $\gamma$ в $\mathbb{C}$, ведуший от регулярного значения $*$ к критическому значению $c$ морсификации $\widetilde{f}$, определяет петлю в $\mathbb{C}$ с началом в точке $*$. Эта петля выходит из $*$ вдоль $\gamma$, подходит к $c$, обходит $c$ один раз в положительном направлении и возвращается в $*$ вдоль $\gamma^{-1}$. Построенная петля назьвается простой. Она определяет автоморфизм Пикара-Лефиеца $h_{\gamma}$ групшы $\bar{H}_{n}(V, \mathbb{Z})$ :

$$
h_{\gamma}: a \mapsto a+(-1)^{(n+1)(n+2) / 2}\langle a, e\rangle e,
$$

где $e$ - цикл, исчезающий вдоль $\gamma, \mathrm{a}\langle\cdot, \cdot\rangle$ - форма пересечений. При четном $n$ преобразование $h_{\gamma}$ является инволюцией, неподвижной на гиперплоскости $\langle a, e\rangle=0$.

ПримеР 1.6. Система путей примера 1.5 определяет два оператора монодромии на $\bar{H}_{0}(V)$, переставляющие $[-\sqrt{3}]$ с $[0]$ и $[0]$ с $[\sqrt{3}]$ соответственно. Следовательно, группа монодромии функции $f=z^{3}$ есть группа Вейля $A_{2}$.

\section{4. Классификация простых особенностей функций.}

ОПРЕДЕЛЕнИЕ 1.7. Два ростка функций $f, g:\left(\mathbb{C}^{n+1}, 0\right) \rightarrow(\mathbb{C}, 0)$ назьваются әквивалентными $(f \sim g)$, если найдется такой росток $b$ биголоморфизма пространства $\left(\mathbb{C}^{n+1}, 0\right)$, что $f=g \circ b$.

ОПРеДЕЛЕниЕ 1.8. Два ростка функций, $f$ и $g$, определенные на различных пространствах $\left(\mathbb{C}^{r}, 0\right)$ и $\left(\mathbb{C}^{s}, 0\right)$, назьваются стабильно әквивалентными, если они становятся эквивалентными после добавления квадратов новых переменных:

$$
f\left(x_{1}, \ldots, x_{r}\right)+x_{r+1}^{2}+\cdots+x_{m}^{2} \sim g\left(y_{1}, \ldots, y_{s}\right)+y_{s+1}^{2}+\cdots+y_{m}^{2}
$$


ОПрЕДЕЛЕнИЕ 1.9. Класс эквивалентности $X$ ростков функций примыкает к классу эквивалентности $Y, X \rightarrow Y$, если представителя класса $X$ можно продеформировать в представителя класса $Y$ сколь угодно малым возмушением.

ОПРЕДЕЛЕНИЕ 1.10. Класс эквивалентности ростка функции назьвается прос$m b l$, если он примыкает только к конечному числу классов эквивалентности.

Теорема 1.11 (В.И. Арнольд [1]). С точностью до стабильной эквивалентности список простых ростков функиий следующий:

\begin{tabular}{|c|c|c|c|c|}
\hline$A_{k}, k \geqslant 0$ & $D_{k}, k \geqslant 4$ & $E_{6}$ & $E_{7}$ & $E_{8}$ \\
\hline$x_{1}^{k+1}$ & $x_{1}^{2} x_{2}+x_{2}^{k-1}$ & $x_{1}^{3}+x_{2}^{4}$ & $x_{1}^{3}+x_{1} x_{2}^{3}$ & $x_{1}^{3}+x_{2}^{5}$ \\
\hline
\end{tabular}

Если число переменных нечетно, группа монодромии простой функции есть соответствующая группа Вейля. Если число переменных сравнимо с 1 по модулю 4, то существует отмеченный базис приведенных гомологий слоя Милнора, в котором матрица пересечений есть матрица Картана соответствующей группь Вейля.

Отметим, что индекс в обозначении простой особенности есть ее число Милнора.

Стабилизация функции добавлением квадратов двух новых переменных приводит к двукратной надстройке каждого элемента отмеченного базиса, что канонически меняет знак пересечения циклов на противоположный [11]. Следовательно, если число переменных сравнимо с 3 по модулю 4, то отмеченньй базис простой особенности может быть выбран так, что матрища пересечений будет матрищей Картана, взятой со знаком минус.

Все возможные примькания выписанных особенностей являются композициями следуюших:

$$
A_{k} \rightarrow A_{k-1}, \quad D_{k} \rightarrow D_{k-1}, A_{k-1}, \quad E_{k} \rightarrow E_{k-1}, D_{k-1}, A_{k-1} .
$$

ЗАмЕчАниЕ 1.12. Мы видим, что простые функции нечетного числа переменных эллиптичны: их форма пересечений невырождена и знакоопределена. Это влечет конечность группы монодромии. Ни одна непростая особенность не обладает такими свойствами.

Еще одно проявление групп Вейля в рассматриваемой ситуации таково.

Пусть $\mathscr{O}_{n+1}-$ пространство всех ростков голоморфных функций на $\left(\mathbb{C}^{n+1}, 0\right)$. Версальная деформация ростка функции $f \in \mathscr{O}_{n+1}$ - эта деформация, содержашая, в некотором смысле, всевозможные деформации $f$. Она называется миниверсальной, если ее база имеет минимальную размерность. Эта минимальная размерность называется коразмерностью функции $f$, она совпадает с числом Милнора. Миниверсальную деформацию можно взять в виде

$$
f+\lambda_{1} e_{1}+\cdots+\lambda_{\mu} e_{\mu},
$$

где элементы $e_{i} \in \mathscr{O}_{n+1}$ образуют базис линейного пространства

$$
\mathscr{O}_{n+1} /\left(\frac{\partial f}{\partial x_{1}}, \ldots, \frac{\partial f}{\partial x_{n+1}}\right)
$$

a $\lambda_{i} \in \mathbb{C}$ являются параметрами деформации [3]. 
Дискриминант ростка $f, \Delta(f) \subset \mathbb{C}^{\mu},-$ это множество таких значений параметров миниверсальной деформации, при которых соответствующая функция на $\mathbb{C}^{n+1}$ имеет нулевое критическое значение.

Если функция $X_{\mu}$ проста, то ее дискриминант $\Delta \subset \mathbb{C}^{\mu}$ биголоморфно эквивалентен множеству нерегулярных орбит в пространстве орбит $\mathbb{C}^{\mu} / X_{\mu} \simeq \mathbb{C}^{\mu}$ комплексификации группы Вейля $X_{\mu}$. Тем самым, дополнение $\mathbb{C}^{\mu} \backslash \Delta$ является пространством Эйленберга-Маклейна $k(\pi, 1)$ для групшы обобшенных кос Брискорна $B\left(X_{\mu}\right)[5]$ (группа обычных кос из $(k+1)$-й нити есть $\left.B\left(A_{k}\right)\right)$. Группа монодромии особенности $X_{\mu}$ есть образ представления групшы $B\left(X_{\mu}\right)$.

Пример 1.13. Миниверсальная деформация особенности $A_{2}$ может быть взята в виде $z^{3}+\lambda_{1} z+\lambda_{2}$. Дискриминант этого семейства есть множество значений $\left(\lambda_{1}, \lambda_{2}\right) \in$ $\mathbb{C}^{2}$, соответствующих полиномам с кратными корнями.

Комплексификация группы Вейля $A_{2}$ - это симметрическая группа трех элементов, действующая в плоскости $\mathbb{C}^{2}=\left\{z_{1}+z_{2}+z_{3}=0\right\} \subset \mathbb{C}^{3}$ перестановками координат. Пространство орбит $\mathbb{C}^{2} / A_{2} \simeq \mathbb{C}^{2}$ отождествляется с пространством полиномов $z^{3}+\lambda_{1} z+\lambda_{2}$. Множество нерегулярных орбит - образ зеркал $z_{i}=z_{j}$, т.е. множество полиномов с кратными корнями.

\section{§. Краевые особенности}

2.1. Исчезающие циклы и полуциклы. Предположим, что в области определения $\left(\mathbb{C}^{n+1}, 0\right)$ ростка $f$ выделена гиперплоскость $\left(\mathbb{C}^{n}, 0\right)$. Будем называть эту гиперплоскость краем. Ограничение $f$ на край обозначим $f_{0}$.

Взяв подходящий шар $B \subset \mathbb{C}^{n+1}$ и малое число $\varepsilon$, рассмотрим пару слоев Милнора

$$
V=\{f=\varepsilon\} \cap B \supset V_{0}=\left\{f_{0}=\varepsilon\right\} \cap\left(B \cap \mathbb{C}^{n}\right) .
$$

Справедливо очевидное

ПРЕДЛОЖЕНИЕ 2.1.

$$
H_{n}\left(V, V_{0} ; \mathbb{Z}\right)=\mathbb{Z}^{\mu+\mu_{0}},
$$

где $\mu$ и $\mu_{0}-$ числа Милнора ростков $f$ и $f_{0}$.

Аналогично абсолютному случаю можно определить отмеченный базис в относительных гомологиях. Для этого возьмем такую морсификацию $\widetilde{f}$ функции $f$, ограничение $\widetilde{f}_{0}$ которой на край есть морсовская функция. Предполагается, что критические значения функций $\widetilde{f}$ и $\widetilde{f}_{0}$ все различны.

Возьмем значение $* \in \mathbb{C}$, регулярное для обеих функций $\widetilde{f}$ и $\widetilde{f}_{0}$. Как и ранее, несамопересекающийся путь в $\mathbb{C}$, идущий из точки $*$ в критическое значение функции $\widetilde{f}$, определяет исчезаюший цикл в $V$ и, тем самым, элемент групшы $H_{n}\left(V, V_{0}\right)$. Путь, идуший в критическое значение функции $\widetilde{f}_{0}$, определяет элемент в $H_{n}\left(V, V_{0}\right)$, называемый исчезающим полуииклом. Локально этот полуцикл представляется множеством

$$
\left\{x_{0}+x_{1}^{2}+\cdots+x_{n}^{2}=\delta>0\right\}
$$

в полупространстве $\left\{x_{0} \geqslant 0\right\}$ вешественного $x$-пространства $\mathbb{R}^{n+1}$ (край задан уравнением $\left.x_{0}=0\right)$. При стремлении $\delta$ к нулю это множество стягивается в точку. 
Как и в абсолютном случае, система из $\mu+\mu_{0}$ путей в $\mathbb{C}$, соединяюших точку $* \mathrm{c}$ критическими значениями функций $\widetilde{f}$ и $\widetilde{f}_{0}$, определяет отмеченный набор исчезаюших циклов и полуциклов, являюшийся базисом в $H_{n}\left(V, V_{0} ; \mathbb{Z}\right)$. Конечно, мы предполагаем, что пути несамопересекаюшиеся и не имеют обших точек, кроме самой точки $*$.

2.2. Двулистное накрытие. Чтобы получить удобную форму пересечений, а вместе с тем и хорошее описание монодромии краевой особенности, Арнольд ввел двулистное накрытие многообразия с краем, разветвленное над краем [2]. Для перехода к такому накрытию положим $x_{0}=z^{2}$. При такой замене функция $f$ преврашается в четную по $z$ функцию $\widehat{f}\left(z, x_{1}, \ldots, x_{n}\right)=f\left(z^{2}, x_{1}, \ldots, x_{n}\right)$. Ее число Милнора равно $\widehat{\mu}=2 \mu+\mu_{0}$.

Слой Милнора $\widehat{V}$ функции $\widehat{f}$ двулистно накрывает слой Милнора $V$ функции $f$, ветвясь над слоем Милнора $V_{0}$ функции $f_{0}$. На $H_{n}(\widehat{V})$ действует инволюция $z \mapsto-z$. Ранг антиинвариантной части $H_{-1} \subset H_{n}(\widehat{V})$ гомологий равен $\mu+\mu_{0}$, т.е. совпадает с рангом группы $H_{n}\left(V, V_{0}\right)$. Пространство $H_{-1}$ заменяет $H_{n}\left(V, V_{0}\right)$ всюду, где используется форма пересечений.

Естественньй базис пространства $H_{-1}$ возникает из базиса пространства $H_{n}\left(V, V_{0} ; \mathbb{Z}\right)$. Он образован длинньми и короткими ииклами, которые есть соответственно полные прообразы (подходяще ориентированные) исчезающих циклов и полуциклов в $\left(V, V_{0}\right)$. Индекс самопересечения короткого цикла совпадает с индексом самопересечения обычного исчезаюшего цикла (1). Индекс самопересечения длинного цикла в два раза больше, т.е. 0,4 или -4 , в зависимости от размерности.

Действующие на $H_{-1}$ и порождающие группу монодромии операторы ПикараЛефшеца задаются формулой (2), если соответствуюший исчезаюший цикл короткий, или же выражением

$$
h_{\gamma}: a \mapsto a+(-1)^{(n+1)(n+2) / 2}\langle a, e\rangle e / 2,
$$

если исчезающий цикл $е$ длинньй.

2.3. Простые краевые особенности. Два ростка, $f$ и $g$, функций на многообразии $\left(\mathbb{C}^{n+1}, 0\right)$ с краем $\left(\mathbb{C}^{n}, 0\right)$ әквивалентны, если найдется такой росток биголоморфного отображения $b$ пары $\left(\mathbb{C}^{n+1}, \mathbb{C}^{n}\right)$, что $f=g \circ b$.

Теорема 2.2 (В.И. Арнольд, [2]). С точностью до стабильной эквивалентности список простых ростков функиий на многообразии $\left(\mathbb{C}^{n+1}, 0\right)=\left\{\left(x_{0}, \ldots, x_{n}\right)\right\}$ с краем $x_{0}=0$ дается следующей таблищей:

\begin{tabular}{|c|c|c|c|}
\hline$A_{k}, D_{k}, E_{k}$ & $B_{k}, k \geqslant 2$ & $C_{k}, k \geqslant 3$ & $F_{4}$ \\
\hline $\begin{array}{c}x_{0}+f_{0}\left(x_{1}, \ldots, x_{n}\right), \\
f_{0} \in A_{k}, D_{k}, E_{k}\end{array}$ & $x_{0}^{k}$ & $x_{0} x_{1}+x_{1}^{k}$ & $x_{0}^{2}+x_{1}^{3}$ \\
\hline
\end{tabular}

Если число переменных нечетно, то группа монодромии простой функиии, действующая на антиинвариантной части гомологий $H_{-1}$, есть одноименная группа Вейля. Если число переменных сравнимо с 1 по модулю 4, то для простой краевой функиии найдется отмеченный базис пространства $H_{-1}$, в котором 
матрица пересечений есть матрица Картана соответствующей әруппь Вейля.

Свойство эллиптичности из замечания 1.12 очевидным образом обобщается на случай краевых особенностей.

В терминах диаграммы Дьнкина группы Вейля двулистное накрытие из предыдущего пункта есть фактор по инволюции канонической диаграммы (рис. 2). В терминах самих групп это соответствует переходу к подгруппе, порожденной произведениями коммутирующих отражений в корнях, склеивающихся при складывании диаграммы.

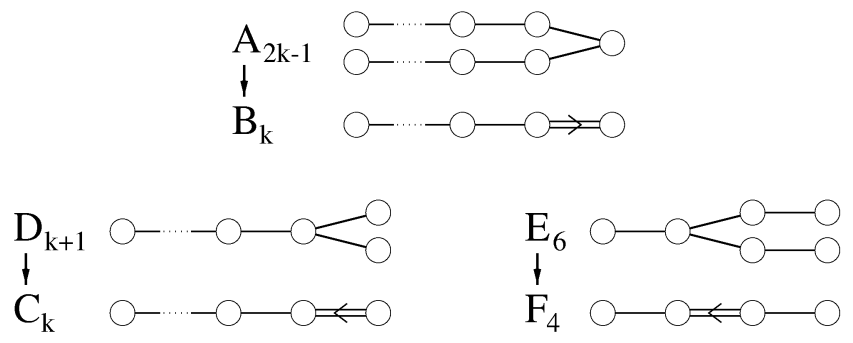

Рис. 2. Складывание диаграмм Дынкина

Все примыкания особенностей теоремы 2.2 соответствуют включениям соответствуюших канонических диаграмм Дьнкина.

Все, что было сказано о связях между дискриминантом функции и множеством нерегулярных орбит отвечающей ей группы Вейля в конце п. 1.4, справедливо и для краевых особенностей, правда, с минимальными оговорками. А именно, миниверсальную деформацию (с базой размерности $\left.\mu+\mu_{0}\right)$ краевой особенности $f \in \mathscr{O}_{n+1}$ можно выбрать в виде

$$
f+\lambda_{1} e_{1}+\cdots+\lambda_{\mu+\mu_{0}} e_{\mu+\mu_{0}},
$$

где ростки $e_{i}$ образуют базис пространства

$$
\mathscr{O}_{n+1} /\left(x_{0} \frac{\partial f}{\partial x_{0}}, \frac{\partial f}{\partial x_{1}}, \ldots, \frac{\partial f}{\partial x_{n}}\right)
$$

Дискриминант функции $f$ теперь состоит из двух частей, отвечающих тем значениям параметра, при которых либо сама функция, либо ее ограничение на край имеют нулевое критическое значение.

\section{§ 3. Конечные группы, порожденные унитарными отражениями}

Список унитарных групп, с которыми мы будем иметь дело, приведен на рис. 3. Он содержит одну двухиндексную серию и семь исключительных групп.

В первом столбце таблицы приведены обозначения груп, восходящие к работе Шепарда и Тодда [19]. Например, индекс в обозначении исключительной группы - это ее номер в классификации [19]. Мы считаем, что в серии $G(m, 1, k)$ у нас $k \geqslant 1$ (число вершин графа) и $m \geqslant 3$ (группа $G(2,1, k)$ есть группа Вейля $\left.B_{k}\right)$. 


\begin{tabular}{|c|c|c|}
\hline $\mathrm{G}(\mathrm{m}, 1, \mathrm{k})$ & $\mathrm{m}=(2)-2$ & $\mathrm{~m}, 2 \mathrm{~m}, \ldots, \mathrm{km}$ \\
\hline $\mathrm{G}_{4}$ & (3) -3 & 4,6 \\
\hline $\mathrm{G}_{5}$ & (3) $=3$ & 6,12 \\
\hline $\mathrm{G}_{8}$ & (4) (4) & 8,12 \\
\hline $\mathrm{G}_{16}$ & (5)-5 & 20,30 \\
\hline $\mathrm{G}_{25}$ & (3)-3 (3) & $6,9,12$ \\
\hline $\mathrm{G}_{26}$ & (2) $\Rightarrow$ (3) (3) & $6,12,18$ \\
\hline $\mathrm{G}_{32}$ & (3)-3) (3) (3) & $12,18,24,30$ \\
\hline
\end{tabular}

Р ис. 3. Соотношения, определяюшие конечные унитарные группы отражений, и степени базисных инвариантов

Последний столбец содержит обозначения тех же групп, которые более удобны с точки зрения теории особенностей (позже мы увидим это), они частично взяты из работы [6]. Нижний индекс равен размерности стандартного линейного представления.

В третьем столбце указаны степени базисных инвариантов стандартного представления. Порядок группы равен произведению этих степеней, поскольку множества орбит групп Шепарда-Тодда гладкие.

Второй столбец содержит диаграммы, кодирующие абстрактные представления групп. Каждая вершина есть образуюшая, порядок которой указан внутри вершины. Кратность ребра, соединяюшего образуюшие $u$ и $v$, на 2 меньше длины соответствуюшего косового соотношения:

$u v=v u$, если вершины не соединены;

$u v u=v u v$, если ребро простое;

$u v u v=v u v u$ для двойного ребра.

Таким образом, любая из наших унитарных групп есть факторгруппа группы обобщенных кос, отвечающей групе Вейля, использованной в обозначениях четвертого столбца таблищы рис. 3. Унитарная группа получается, если образующим приписать конечные поря дки. Приписав всем образуюшим порядок 2, мы вернемся к самой группе Вейля.

Точное значение знака неравенства на двойном ребре диаграммы мы объясним позже, в п. 5.2. Там диаграмма представления будет проинтерпретирована как диаграмма Дынкина унитарного аналога системы корней группы Вейля. Как и в евклидовом случае, знак неравенства указывает на разницу в длинах корней.

Отметим, что серия $G(m, 1, k)$ содержит, при $k=1$, все конечные циклические группы, занимающие третью строку в списке статьи [19]. 
Стандартное линейное представление групшы может быть получено из ее графа следующим образом [9], [8]. Рассмотрим векторное пространство $\mathbb{C}^{n}$, порожденное вершинами $v_{1}, \ldots, v_{n}$ графа. Граф задает положительно определенную эрмитову форму $\langle\cdot, \cdot\rangle$ на $\mathbb{C}^{n}$ :

$$
\begin{aligned}
& \left\langle v_{i}, v_{i}\right\rangle=\sin \frac{\pi}{p_{i}}, \text { где } p_{i}-\text { число в вершине (порядок образуюшей); } \\
& \left\langle v_{i}, v_{j}\right\rangle=0, \text { если вершины не соединены ребром; } \\
& \left\langle v_{i}, v_{j}\right\rangle=-\cos \frac{\pi}{3}=-\frac{1}{2} \text { для простого ребра; } \\
& \left\langle v_{i}, v_{j}\right\rangle=-\left(\frac{1}{2} \sin \frac{\pi}{p_{j}}\right)^{1 / 2} \text { для двойного ребра с } p_{i}=2 ; \\
& \left\langle v_{1}, v_{2}\right\rangle=-\cos \frac{\pi}{4}=-\frac{1}{\sqrt{2}} \text { для } G_{5} .
\end{aligned}
$$

Соответствуюшая вершине образуюшая (комплексное отражение) есть вращение порядка $p_{j}$ вокруг гиперплоскости, эрмитово-ортогональной вектору $v_{j}$ :

$$
v \mapsto v+\left(e^{2 \pi i / p_{j}}-1\right) \frac{\left\langle v, v_{j}\right\rangle}{\left\langle v_{j}, v_{j}\right\rangle} v_{j}
$$

\section{§ 4. Циклическое накрытие функции на многообразии с краем}

Мы возврашаемся к краевьм особенностям п. 2.1, сохраняя все обозначения. Обобщая накрывающую конструкцию п. 2.2 , положим

$$
x_{0}=z^{m},
$$

где $m$ - произвольное целое число, большее 1 . Мы получаем $m$-листное накрытие $c: \widehat{\mathbb{C}}^{n+1} \rightarrow \mathbb{C}^{n+1}$, разветвленное над краем.

Как и ранее, функция $f$ на многообразии $\mathbb{C}^{n+1}$ с границей $\mathbb{C}^{n}$ поднимается до функции $\widehat{f}=f \circ c$ на $\widehat{\mathbb{C}}^{n+1}$. На этот раз

$$
\widehat{\mu}=m \mu+(m-1) \mu_{0} .
$$

Возьмем прообраз слоя Милнора функции $f$ в качестве слоя Милнора функции $\widehat{f}$, $\widehat{V}=c^{-1}(V)$. Группа $\mathbb{Z}_{m}$ накрытия $c$ действует в слое $\widehat{V}$ и на его гомологиях. Мы получаем разложение

$$
\bar{H}_{n}(\widehat{V}, \mathbb{C})=\bigoplus_{\chi^{m}=1} H_{\chi}
$$

в подпространства, на каждом из которых $\mathbb{Z}_{m}$ действует умножением на характер $\chi$. Будем назьвать эти подпространства характеристическими. Очевидно, что

$$
\operatorname{dim} H_{\chi=1}=\mu, \quad \operatorname{dim} H_{\chi \neq 1}=\mu+\mu_{0} .
$$

Геометрический базис подпространства $H_{\chi}$ может быть получен из отмеченного базиса исчезающих циклов и полуциклов краевой пары слоев Милнора $\left(V, V_{0}\right)$ следующим образом (см. рис. 4).

Полньй прообраз исчезающего цикла $\sigma$ при отображении $c$ состоит из $m$ циклов $\sigma_{0}, \ldots, \sigma_{m-1}$ в $\widehat{V}$. Возьмем их с ориентацией, поднятой с $\sigma$. Будем считать, что индексация циклов $\sigma_{j}$ выбрана так, что врашение $z \mapsto e^{2 \pi i / m} z$ дает циклическую перестановку $\sigma_{j} \mapsto \sigma_{(j+1)} \bmod m$. Линейная комбинация

$$
\sigma_{\chi}=\sum_{j=0}^{m-1} \chi^{-j} \sigma_{j}
$$



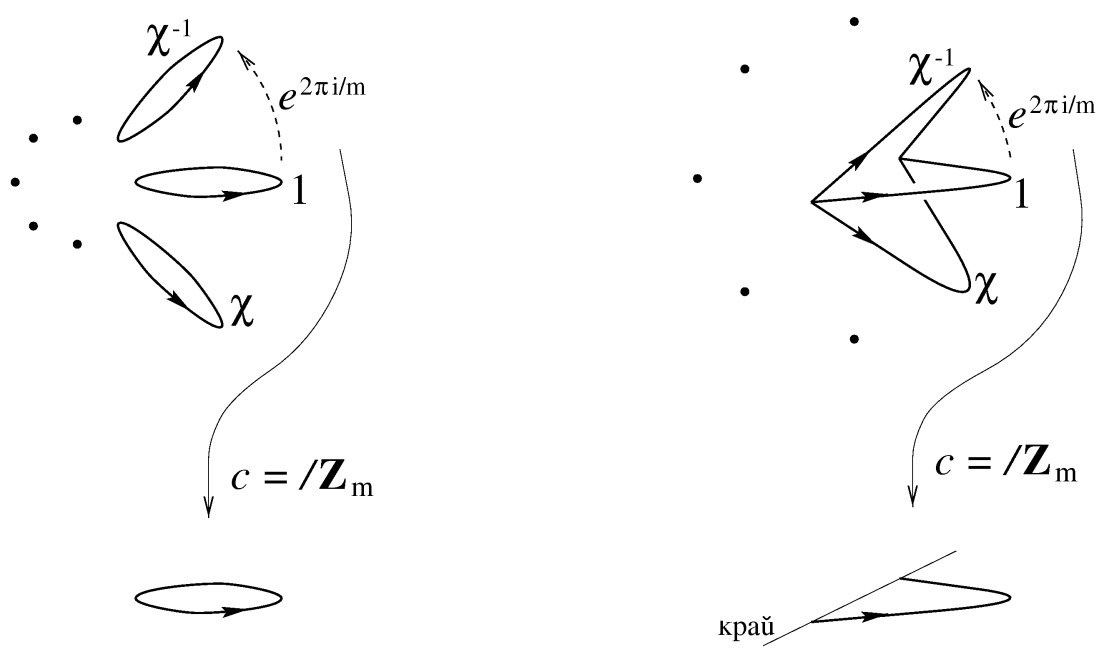

Р ис. 4. Поднятие цикла и полуцикла краевой особенности $f$ до длинных и коротких $\chi$-циклов в характеристическомподпространстве $H_{\chi} \mathbb{Z}_{m}$-симметричной функции $\widehat{f}=f \circ c$

определяет ненулевой элемент в $H_{\chi}$. Назовем его длинным $\chi$-ииклом.

Для исчезающего полуцикла в $\left(V, V_{0}\right)$ составим аналогичную линейную комбинацию его $m$ прообразов в $\widehat{V}$. Эта линейная комбинация является циклом, если $\chi \neq 1$ $\left(\right.$ поскольку $\left.1+\chi+\cdots+\chi^{m-1}=0\right)$, и определяет ненулевой элемент в $H_{\chi}$. Назовем этот элемент коротким ұ-циклом.

Как длинные, так и короткие $\chi$-циклы определены с точностью до умножения на степени $\chi$ и на -1 .

Рассмотрим теперь (косо-)эрмитову форму на пространстве $\bar{H}_{n}(\widehat{V}, \mathbb{C})$, задаваемую индексом пересечения $\langle\cdot, \cdot\rangle$.

ПРЕДЛОЖЕНИЕ 4.1. Индекс самопересечения длинного ұ-иикла равен нулю, если п нечетно, и $(-1)^{n / 2} 2 m$ при четном $n$. Индекс самопересечения короткого $\chi$-иикла равен соответственно $(-1)^{(n-1) / 2} \frac{1+\chi}{1-\chi} m u(-1)^{n / 2} m$.

Это очевидно для длинных циклов. В случае коротких циклов утверждение основано на следующей лемме.

Лемма 4.2. Рассмотрим локальное пересечение общего положсения двух полуциклов на $V$ в точке подмногообразия $V_{0}$. Можсно так выбрать соответствующие короткие $\chi$-иикль на $\widehat{V}$, что их локальный индекс пересечения составит $m /(1-\chi)$.

Требуемое локальное поднятие полуциклов показано на рис. 5 . Из рис. 6 следует, что локальное пересечение именно такое, как утверждается в лемме.

Конструкция группы монодромии краевой особенности дает операторы ПикараЛефшеца $h_{\gamma}: \bar{H}_{n}(\widehat{V}, \mathbb{C}) \rightarrow \bar{H}_{n}(\widehat{V}, \mathbb{C})$. Каждый из них распадается в прямую сумму $h_{\gamma}=\bigoplus_{\chi^{m}=1} h_{\gamma, \chi}$ операторов, действующих на индивидуальных характеристических подпространствах $H_{\chi}$. 


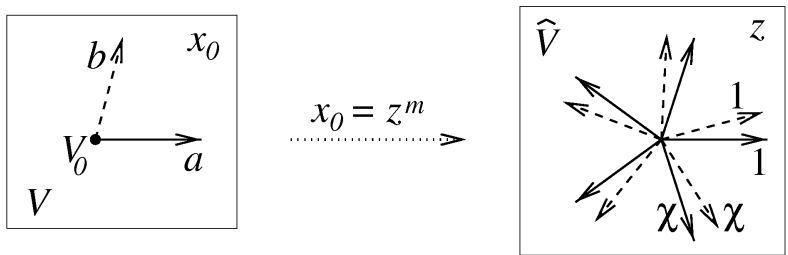

Рис. 5. Выбор локального поднятия пересечения двух полуциклов в пространство $H_{\chi}$

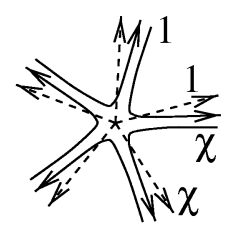

Рис. 6. Доказательство того, что для поднятых циклов предыдущего рисунка $\langle(1-\chi) \widehat{a}, \widehat{b}\rangle=m$

ПРЕДЛОЖЕНИЕ 4.3. Действие оператора Пикара-Лефшеца $h_{\gamma, \chi}: H_{\chi} \rightarrow H_{\chi}$, отвечающего короткому исчезающему ұ-ииклу е, задается формулой

$$
h_{\gamma, \chi}: a \mapsto a+(-1)^{(n+1)(n+2) / 2}(1-\chi)\langle a, e\rangle e / m .
$$

Аналогичный оператор, отвечающий длинному исчезающему ұ-циклу, есть

$$
h_{\gamma, \chi}: a \mapsto a+(-1)^{(n+1)(n+2) / 2}\langle a, e\rangle e / m .
$$

Таким образом, в симметричном случае ( $n$ четно) операторы Пикара-Лефшеца являются врашениями вокруг гиперплоскостей, эрмитово-ортогональных соответствующим циклам. Их порядки равны соответственно порядку $\chi$ и двойке.

ПримеР 4.4. Рассмотрим накрытие $x_{0}=z^{m}$ деформации $f(x)=x^{2}-4 x+3$ одномерной краевой особенности $B_{2}$. Возьмем $\widehat{f}=0$ в качестве накрьвающего слоя Милнора $\widehat{V}$ (см. рис. 7). Соединим точку $0 \in \mathbb{C}$ прямьми путями с критическими значениями $f(0)=3$ и $f(2)=-1$ функции $f$. Возьмем линейную комбинацию точек на внутренней окружности рис. 7 в качестве короткого $\chi$-цикла $e_{1} \in H_{\chi} \subset \bar{H}_{0}(\widehat{V})$, исчезающего на уровне $\widehat{f}=3$, и разность между линейными комбинациями точек на внутренней и внешней окружностях в качестве длинного $\chi$-цикла $e_{2}$, исчезаюшего на уровне $\widehat{f}=-1$. Матрица пересечений $\left(\left\langle e_{i}, e_{j}\right\rangle\right)$ есть

$$
\left(\begin{array}{cc}
m & -m \\
-m & 2 m
\end{array}\right) \text {. }
$$

Оператор Пикара-Лефшеца $h_{1}$ врашает точки на внутренней окружности против часовой стрелки на угол $2 \pi / m$, что означает

$$
\begin{aligned}
& h_{1}\left(e_{1}\right)=\chi e_{1} \\
& h_{1}\left(e_{2}\right)=e_{2}+(1-\chi) e_{1}=e_{2}-(1-\chi)\left\langle e_{2}, e_{1}\right\rangle e_{1} / m=e_{2}+(\chi-1) \frac{\left\langle e_{2}, e_{1}\right\rangle}{\left\langle e_{1}, e_{1}\right\rangle} e_{1} .
\end{aligned}
$$




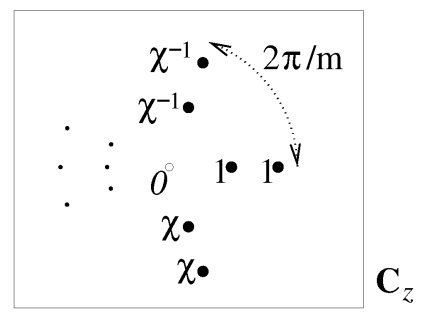

Рис. 7. Исчезающие $\chi$-циклы для накрытия степени $m$ особенности $B_{2}$

Оператор $h_{2}$ меняет местами точки на каждом луче, выходящем из начала координат:

$$
\begin{aligned}
& h_{2}\left(e_{1}\right)=e_{1}+e_{2}=e_{1}-\left\langle e_{1}, e_{2}\right\rangle e_{2} / m=e_{1}+\left(e^{2 \pi i / 2}-1\right) \frac{\left\langle e_{1}, e_{2}\right\rangle}{\left\langle e_{2}, e_{2}\right\rangle} e_{2}, \\
& h_{2}\left(e_{2}\right)=-e_{2} .
\end{aligned}
$$

Оба оператора дают наглядное представление о предложении $4.3(n=0)$. Сравнивая выписанные выше формулы с (4) и определениями $\S 3$, мы видим, что операторы $h_{1}$ и $h_{2}$ порождают в $U(2)$ подгруппу, изоморфную $B_{2}^{(p)}$, где $p$ - порядок $\chi$. В самом деле, порядки образуюших совпадают с порядками канонических порождающих отражений групшы $B_{2}^{(p)}$, а матрица пересечений в отнормированном базисе $\left\{e_{1} / \sqrt{m \sin \frac{\pi}{p}}, e_{2} / \sqrt{2 m \sin \frac{\pi}{p}}\right\}-$ в точности та же, что и определяемая графом $B_{2}^{(p)}$. Отметим также, что, коль скоро косовое соотношение выполняется для образуюших группы отражений, то оно выполняется и для их степеней.

В следуюшем параграфе мы обобщаем эти наблюдения на случай всех рассматриваемых нами унитарных групп отражений.

\section{$\S$ 5. Функции с циклической симметрией}

5.1. Эллиптические особенности. Группа $\mathbb{Z}_{m}$ накрытия $c: \widehat{\mathbb{C}}^{n+1} \rightarrow \mathbb{C}^{n+1}$ из предыдущего параграфа действует на пространстве $\widehat{\mathbb{C}}^{n+1}$ унитарными отражениями, которые неподвижны на множестве ветвления $\mathbb{C}^{n}$. Забудем на время о накрытии и рассмотрим ростки голоморфных функций на $\left(\widehat{\mathbb{C}}^{n+1}, 0\right)$, инвариантные относительно этого действия. Назовем их функииями с ииклической симметрией или просто ииклическими особенностями.

Естественно считать такие функции эквивалентными, если они переводятся друг в друга $\mathbb{Z}_{m}$-эквивариантньми биголоморфизмами пространства $\left(\widehat{\mathbb{C}}^{n+1}, 0\right)$. Конечно, это просто краевая эквивалентность функций, опущенных на факторпространство $\left(\widehat{\mathbb{C}}^{n+1}, 0\right) / \mathbb{Z}_{m}$, базу нашего накрытия $c$. Стабильная эквивалентность добавляет квадраты новых переменных, на которые наша группа симметрий действует тривиально.

(Мини)версальная деформация циклической особенности в пространстве функций с той же симметрией индуцируется накрытием $c$ из (мини)версальной деформации соответствуюшей функции на многообразии с краем. Размерность базы миниверсальной деформации циклической особенности, т.е. ее коразмерность, равна $\mu+\mu_{0}$. 
В качестве группь монодромии ииклической особенности возьмем группу, порожденную операторами Пикара-Лефшеца $h_{\gamma}=\bigoplus_{\chi} h_{\gamma, \chi}$ из предыдущего параграda.

ОПРЕДЕЛЕНИЕ 5.1. Функция с циклической симметрией назьвается әллиптической, если она стабильно эквивалентна циклической особенности нечетного числа переменных с конечной группой монодромии.

Классификация эллиптических циклических особенностей более содержательна, нежели классификация простых циклических особенностей. В последнем случае мы получаем более длинньй и совершенно очевидньй список, состояший из произвольных циклических поднятий простых краевых особенностей.

С точностью до стабильной эквивалентности эллиптические циклические особенности с $\mathbb{Z}_{2}$-симметрией есть двулистные накрытия простых краевых особенностей. Для высшей симметрии имеет место

TеОРема 5.2. С точностью до стабильной әквивалентности список әллиптических функиий с ииклической симметрией $\mathbb{Z}_{m}, m>2$, таков:

\begin{tabular}{|c|c|c|c|}
\hline обозначение & $\begin{array}{c}\text { соответствующая } \\
\text { краевая } \\
\text { особенность }\end{array}$ & $\begin{array}{c}\text { порядок } \\
\text { накрытия } \\
x_{0}=z^{m}\end{array}$ & $\begin{array}{c}\text { накрывающая } \\
\text { абсолютная } \\
\text { особенность }\end{array}$ \\
\hline$B_{k}^{(m)}$ & $B_{k}: x_{0}^{k}, k \geqslant 1$ & $m$ & $A_{k m-1}$ \\
$A_{2}^{(3)}$ & $A_{2}: x_{0}+x_{1}^{3}$ & 3 & $D_{4}$ \\
$A_{2}^{(4)}$ & $A_{2}: x_{0}+x_{1}^{3}$ & 4 & $E_{6}$ \\
$A_{2}^{(5)}$ & $A_{2}: x_{0}+x_{1}^{3}$ & 5 & $E_{8}$ \\
$A_{3}^{(3)}$ & $A_{3}: x_{0}+x_{1}^{4}$ & 3 & $E_{6}$ \\
$C_{3}^{(3)}$ & $C_{3}: x_{0} x_{1}+x_{1}^{3}$ & 3 & $E_{7}$ \\
$A_{4}^{(3)}$ & $A_{4}: x_{0}+x_{1}^{5}$ & 3 & $E_{8}$ \\
\hline
\end{tabular}

В случае нечетного числа переменных группа монодромии, действующая на пространстве $H_{\chi}$, әде $\chi$ - примитивный корень степени $m$ из единицы, есть унитарная группа отражений, одноименная с ииклической особенностью.

Размерность характеристического подпространства $H_{\chi \neq 1}$ равна коразмерности соответствуюшей краевой особенности. Для удобства мы считаем, что $B_{1}=A_{1}$.

Особенности примькают очевидным образом (ср. пा. 1.4 и 2.3).

Мы доказываем теорему 5.2 в следующих двух пунктах.

ЗАмечАния 5.3. Нетрудно сделать ряд элементарных, но полезных наблюдений о функциях в таблище: 
(а) Множество степеней базисных инвариантов унитарной групшы отражений, отвечающей эллиптической циклической особенности, является подмножеством аналогичного множества для группы Вейля, указанной в последнем столбце. При этом максимальные степени, т.е. числа Кокстера двух групп, совпадают. Это показывает, как пространства орбит (служашие также базами соответствующих миниверсальных деформаций) вложены друг в друга.

(b) Множество степеней базисных инвариантов унитарной группы $X^{(m)}$ кратно аналогичному множеству группы Вейля $X$. Согласно [16] обе пары \{пространство орбит, множество нерегулярных орбит \} изоморфны (как и ранее, мы рассматриваем здесь комплексификацию канонического представления группы $X)$.

(c) Указанньй изоморфизм - это в точности изоморфизм (доставляемьй накрытием c) между базами миниверсальных деформаций циклической и краевой особенностей. В рамках этой интерпретации множества нерегулярных орбит являются дискриминантами особенностей. Дискриминант ииклической особенности есть множество тех значений параметров деформации, для которых соответствующие функции имеют критическое значение 0.

(d) Как мы уже отмечали, множество регулярных орбит групшы Вейля $X$ является пространством $k(\pi, 1)$ для группы $B(X)$ обобщенных кос. Ее образующие и косовые соотношения между ними читаются по диаграмме Дынкина групшы $X$ так же, как это было указано в $\S 3$, но без требования конечности порядков образующих. Группа монодромии краевой особенности $X$ является представлением групшы $B(X)$, что дает в случае четномерного слоя Милнора саму группу $X$ (квадрат каждой образующей приравнивается к единице группы). Аналогично этому, утверждение о монодромии в теореме говорит, что представление группы $B(X)$ на характеристическом подпространстве $H_{\chi}$ циклической особенности $X^{(m)}$ есть унитарная группа $X^{(m)}$ : оно приравнивает к единище в точности нужные степени образующих и не вводит никаких других соотношений.

5.2. Диаграммы пересечений. Приступим к доказательству теоремы. Покажем прежде всего, что группы монодромии циклических особенностей именно те, что указаны в теореме.

Укажем, каким образом диаграммы рис. 3 можно трактовать как диаграммы Дьнкина отмеченных базисов длинных и коротких $\chi$-циклов пространства $H_{\chi}$, операторы Пикара-Лефшеца которых порождают нужные унитарные группы отражений.

Если опустить числа в вершинах диаграмм рис. 3, то получатся стандартные диаграммы Дынкина систем корней $B_{k}, A_{k}, C_{3}$, с традиционными соглашениями: вершина есть базисный корень; длинные корни имеют длину 2 , а короткие длину $\sqrt{2}$; отсутствие ребра между двумя вершинами означает ортогональность корней; простое ребро показывает, что скалярное произведение корней равно -1 , если оба корня короткие, или -2 , если оба длинные; двойное ребро между длинным и коротким корнями означает, что их произведение равно - 2; ребра, соединяюшие корни разной длины, снабжаются знаком неравенства, открытым в сторону длинного корня.

Каждая простая краевая функция $X$ на $\mathbb{C}^{4 r+1}$ обладает отмеченным базисом циклов и полуциклов пространства $H_{4 r}\left(V, V_{0}\right)$, которьй поднимается на двулистное накрытие в базис длинных и коротких циклов пространства $H_{\chi=-1}$, имеющий стандартную диаграмму Дынкина системы корней $X$ в качестве диаграммы пересечений (цик- 
лы являются корнями, а скалярное произведение задается пересечением в гомологиях). Более того, такой базис в $H_{-1}$ имеет геометрическую реализацию, в которой число точек пересечения двух циклов на $\widehat{V}$ равно абсолютной величине их индекса пересечения. Операторы Пикара-Лефшеца на пространстве $H_{-1}$, отвечающие элементам этого базиса, есть евклидовы отражения в рассматриваемых корнях и порождают группу Вейля $X$.

Стабильная эквивалентность надстраивает описанньй базис относительных гомологий до базиса простой краевой особенности произвольной размерности. Получающийся таким образом базис мы будем назьвать стандартным.

Рассмотрим теперь стандартньй базис краевой особенности и поднимем его на $m$-листное накрытие в отмеченньй базис подпространства $H_{\chi \neq 1}$. Сказанное о пересечениях в этом подпространстве в конце п. 4 влечет (в качестве обобшения вьшеизложенного), что каждая диаграмма рис. 3 (за исключением $G_{5}=B_{2}^{(3,3)}$ ) может трактоваться как диаграмма пересечений получаемого базиса в рамках следуюших соглашений:

- вершина изображает элемент базиса;

- вершине, отвечаюшей длинному $\chi$-циклу, приписьвается число 2, а вершпне, отвечающей короткому $\chi$-циклу, - порядок $\chi$;

- квадраты базисных $\chi$-циклов задаются предложением 4.1;

- отсутствие ребра между вершинами показьвает, что циклы не пересекаются;

- простое ребро, соединяюшее две вершины, означает, что индекс пересечения циклов равен $m /(\chi-1)$, если оба они короткие, или $-m$, если они длинные (при нечетном $n$ формально мы должны фиксировать порядок циклов в пересечении, но в рассматриваемых случаях это не имеет никакого значения, поскольку все наши графы - деревья);

- двойное ребро между циклами разной длины означает, что их индекс пересечения равен $-m$;

- такое ребро снабжается знаком неравенства, открытым в сторону длинного цикла.

Пусть теперь $\chi$ - примитивньй корень степени $m$ из единицы. Легко проверить (ср. пример 4.4), что при четном $n$ можно домножить элементы стандартного базиса подпространства $H_{\chi}$ циклической особенности из таблищы теоремы 5.2 на подходящие комплексные числа так, что полученные циклы будут удовлетворять нормировке, указанной в конце $\S 3$. Тем самым, операторы Пикара-Лефшеца $h_{\gamma, \chi}$ из $\S 4$ действительно порождают обещанную конечную унитарную группу, действующую на $H_{\chi}$.

В случаях $B_{k}^{(m)}$ и $A_{2}^{(4)}$ характер $\chi$ может не быть примитивным. Возникающие тогда группы монодромии есть $B_{k}^{(\text {ord } \chi)}$ и $A_{2}$.

5.3. Окончание доказательства теоремы 5.2. Покажем теперь, что список эллиптических циклических особенностей теоремы 5.2 полон.

Рассмотрим, к примеру, ростки функций с $\mathbb{Z}_{3}$-симметрией. Любая такая функция, не содержащаяся в таблице, примыкает, по крайней мере, к одной из функций $A_{5}^{(3)}$, $D_{4}^{(3)}, C_{4}^{(3)}$ или $F_{4}^{(3)}$ (в понятных обозначениях). Если число переменных нечетно, то группа монодромии каждой из этих четырех особенностей, действующая на подпространстве $H_{\chi \neq 1}$, порождена комплексньпи отражениями, удовлетворяющими соот- 
ношениям, задаваемым соответствующей модифищированной канонической диаграммой Дьнкина (ср. рис. 3), вершины которой имеют порядки 2 (если корень соответствующей групшы Вейля длинньй) и 3 (если корень короткий). Ни одна из четырех таким образом получаемых групп не значится в классификации Шепарда-Тодда.

Для симметрий высшего порядка надо рассмотреть аналогично функции $A_{3}^{(4)}, A_{3}^{(5)}$, $C_{3}^{(>3)}$ и $A_{2}^{(>5)}$.

5.4. Особенность $B_{2}^{(3,3)}$. Соответствие теоремы 5.2 распространяется и на последнюю из унитарных групп отражений, $G_{5}=B_{2}^{(3,3)}$, перечисленных на рис. 3. Чтобы показать это, следует рассмотреть угловые особенности функций на $\mathbb{C}^{n+1}$ (т.е. особенности в присутствии пары гладких гиперповерхностей, трансверсальных друг другу [20]) и циклически накрыть $\mathbb{C}^{n+1}$ дважды, с ветвлением вдоль каждой из гиперповерхностей. Накрытие надо взять с группой $\mathbb{Z}_{3} \times \mathbb{Z}_{2}$ и поднять на него следует единственную угловую особенность коразмерности 2 :

$$
x_{0}+x_{1}^{2}+x_{2}^{2}+\cdots+x_{n}^{2}, \quad x_{0}=z^{3}, \quad x_{1}=w^{2} .
$$

В результате эта угловая особенность “вкладьвается" в $E_{6}$.

Пространство исчезающих гомологий, ранга 6 , симметричной особенности $E_{6}$ распадается в прямую сумму $\bigoplus_{\chi_{0}^{3}=1, \chi_{1}^{2}=1} H_{\chi_{0}, \chi_{1}}$ характеристических подпространств групшы $\mathbb{Z}_{3} \times \mathbb{Z}_{2}$ (на самом деле $H_{1, \pm 1}=0$ ). Геометрический базис в каждом из них можно построить из геометрического базиса подпространства $H_{\chi_{0}}$ групшы $A_{3}^{(3)}$, который вдобавок еще $\mathbb{Z}_{2}$-симметричен или антисимметричен.

Комбинируя таким образом подходы $\S 4$ и п. 2.2, мы получаем для двух двумерных подпространств $H_{e^{ \pm 2 \pi i / 3},-1}$ базисы, состоящие из одного длинного и одного короткого цикла каждый. Индекс самопересечения короткого цикла дается предложением 4.1 $\left(\chi=\chi_{0}\right)$, а у длинного цикла он в два раза больше (в отличие от предложения 4.1 он ненулевой, когда милноровский слой нечетномерен). Короткий и длинньй циклы можно выбрать так, что их индекс пересечения составит $6 /(\chi-1)$. В результате граф $B_{2}^{(3,3)}$ на рис. 3 интерпретируется как диаграмма пересечений в указанном базисе.

Оператор Пикара-Лефшеца, отвечаюший короткому циклу, действует на $H_{\chi,-1}$ по формуле предложения $4.3(m=3)$. Оператор, отвечающий длинному циклу, действует по похожей формуле, но с $2 m=6$ в знаменателе (т.е. отличается от операторов предложения 4.3, соответствующих длинным $\chi$-циклам). Оба оператора имеют порядок 3.

Умножая описанные циклы на числа, можно привести матрицу пересечений к виду, указанному в $\S 3$. Таким образом, при четном $n$ группа монодромии, действующая на каждом из подпространств $H_{e^{ \pm 2 \pi i / 3},-1}$, есть $G_{5}=B_{2}^{(3,3)}$.

Рассмотренную особенность естественно обозначить $B_{2}^{(3,3)}$. Она оказьвается единственной эллиптической особенностью коразмерности вьше 1 с аналогичньм действием произведения циклических групп при условии, что порядок произведения больше 4.

5.5. Целочисленность. Отмеченные базисы подпространств $H_{\chi}$ эллиптических циклических особенностей обладают решеточным свойством, похожим на имеющееся у корневых систем групп Вейля. А именно, образ элемента $а$ такого базиса при 


\begin{tabular}{|c|c|c|}
\hline граф & $\begin{array}{c}\text { матрица пересечений и } \\
\text { операторы монодромии } \\
h_{1}, h_{2} \text { при } n \equiv 0(\bmod 4)\end{array}$ & $\begin{array}{c}\text { матрица пересечений и } \\
\text { операторы монодромии } \\
h_{1}, h_{2} \text { при } n \equiv 1(\bmod 4)\end{array}$ \\
\hline$A_{2}^{(m)}$ & $\begin{array}{l}\left(\begin{array}{cc}m & -\frac{m}{1-\chi} \\
-\frac{m}{1-\bar{\chi}} & m\end{array}\right) \\
\left(\begin{array}{cc}\chi & -\chi \\
0 & 1\end{array}\right)\left(\begin{array}{ll}1 & 0 \\
1 & \chi\end{array}\right)\end{array}$ & $\begin{array}{c}\left(\begin{array}{cc}m \frac{1+\chi}{1-\chi} & -\frac{m}{1-\chi} \\
\frac{m}{1-\bar{\chi}} & m \frac{1+\chi}{1-\chi}\end{array}\right) \\
\left(\begin{array}{cc}-\chi & \chi \\
0 & 1\end{array}\right) \\
\left(\begin{array}{cc}1 & 0 \\
1 & -\chi\end{array}\right)\end{array}$ \\
\hline$B_{2}^{(m)}$ & $\begin{array}{c}\left(\begin{array}{cc}m & -m \\
-m & 2 m\end{array}\right) \\
\left(\begin{array}{cc}\chi & 1-\chi \\
0 & 1\end{array}\right)\left(\begin{array}{cc}1 & 0 \\
1 & -1\end{array}\right)\end{array}$ & $\begin{array}{c}\left(\begin{array}{cc}m \frac{1+\chi}{1-\chi} & -m \\
m & 0\end{array}\right) \\
\left(\begin{array}{cc}-\chi & \chi-1 \\
0 & 1\end{array}\right)\left(\begin{array}{ll}1 & 0 \\
1 & 1\end{array}\right)\end{array}$ \\
\hline $\begin{array}{c}A_{2} \\
\text { длинные } \\
\text { циклы }\end{array}$ & $\begin{array}{c}\left(\begin{array}{cc}2 m & -m \\
-m & 2 m\end{array}\right) \\
\left(\begin{array}{cc}-1 & 1 \\
0 & 1\end{array}\right) \quad\left(\begin{array}{cc}1 & 0 \\
1 & -1\end{array}\right)\end{array}$ & $\begin{array}{c}\left(\begin{array}{cc}0 & -m \\
m & 0\end{array}\right) \\
\left(\begin{array}{cc}1 & -1 \\
0 & 1\end{array}\right)\left(\begin{array}{ll}1 & 0 \\
1 & 1\end{array}\right)\end{array}$ \\
\hline$B_{2}^{(3,3)}$ & $\begin{array}{c}\left(\begin{array}{cc}3 & -\frac{6}{1-\chi} \\
-\frac{6}{1-\bar{\chi}} & 6\end{array}\right) \\
\left(\begin{array}{cc}\chi & -2 \chi \\
0 & 1\end{array}\right)\left(\begin{array}{ll}1 & 0 \\
1 & \chi\end{array}\right)\end{array}$ & $\begin{array}{c}\left(\begin{array}{cc}3 \frac{1+\chi}{1-\chi} & -\frac{6}{1-\chi} \\
\frac{6}{1-\bar{\chi}} & 6 \frac{1+\chi}{1-\chi}\end{array}\right) \\
\left(\begin{array}{cc}-\chi & 2 \chi \\
0 & 1\end{array}\right) \\
\left(\begin{array}{cc}1 & 0 \\
1 & -\chi\end{array}\right)\end{array}$ \\
\hline
\end{tabular}

унитарном отражении в элементе $b$ равен $a+\ell(\chi) b$, где $\ell(\chi)$ есть линейная функция характера с цельми коэффициентами. Коэффициентыне зависят от $\chi$ : если элементы $a, b \in H_{\chi}$ и $a^{\prime}, b^{\prime} \in H_{\chi^{\prime}}$ являются подходяшими поднятиями одних и тех же исчезающих (полу)циклов с $\left(V, V_{0}\right)$, то отражение в $b^{\prime}$ отправляет $a^{\prime}$ в $a^{\prime}+\ell\left(\chi^{\prime}\right) b^{\prime}$.

То же самое наблюдение справедливо и для косо-эрмитовых аналогов рассматриваемых групп.

Указанная целочисленность следует из приводимой на этой странице таблицы, в которой выписаны неприводимые двумерные подгрупшы, порождаемые всевозможными парами $e_{1}, e_{2} \in H_{\chi}$ элементов стандартных отмеченных базисов эллиптических циклических особенностей как в четном, так и в нечетном случаях. При этом мы выписываем и соответствующие матрицы пересечений.

Из строки $A_{2}^{(m)}$ таблишы следует, что монодромия особенности $A_{k}^{(m)}$ есть представление Бюрау группы кос из $(k+1)$-й нити в корнях из единицы степени $m$ (или же в этих корнях, взятых со знаком минус, - в нечетном случае).

Стабильная эквивалентность особенностей, добавляющая две новые переменные, умножает матрицу пересечений на -1 и сохраняет операторы Пикара-Лефшеца. Тем самым, наша таблица дает информацию также и в случаях $n \equiv 2,3(\bmod 4)$. 
5.6. Стягивание диаграмм. Версальная циклическая деформация циклической особенности является поддеформацией в версальной деформации соответствующей абсолютной особенности (например, $B_{k}^{(m)}$ в $A_{k m-1}$ ). Согласно этому отвечающие паре таких особенностей группы монодромии вложены одна в другую. Для $\mathbb{Z}_{2}$-симметричных особенностей это вложение интерпретируется как складьвание канонических диаграмм Дынкина (см. рис. 2). На случай высшей симметрии эта операция обобщается следующим образом.

При элементарном вырождении $B_{1}^{(m)} \mathbb{Z}_{m}$-симметричной функции исчезает один короткий $\chi$-цикл. В этот момент абсолютная особенность имеет критическую точку типа $A_{m-1}$. Тем самым, действие оператора Пикара-Лефшеца $h=\bigoplus_{\chi^{m}=1} h_{\chi}$ на гомологиях симметричного слоя Милнора есть действие оператора классической монодромии особенности $A_{m-1}$ (т.е. взятого в правильном порядке произведения $(m-1)$-го оператора Пикара-Лефшеца несимметричной морсификации этой функции). Короткий $\chi$-цикл является собственным вектором, отвечающим собственному значению $\chi$ рассматриваемого оператора классической монодромии на линейном пространстве, порожденном нашим $(m-1)$-м несимметричным исчезающим циклом.

Аналогично, оператор Пикара-Лефшеца $h=\bigoplus_{\chi^{m}=1} h_{\chi}$, отвечающий $m$ длинным $\chi$-циклам, исчезаюшим на одном критическом уровне $\mathbb{Z}_{m}$-симметричного возмушения циклической особенности, есть произведение $m$ коммутирующих несимметричных операторов Пикара-Лефшеца. Линейное подпространство в гомологиях, порожденное $m$ длинньми $\chi$-циклами, совпадает с подпространством, порожденным соответствуюшими $m$ несимметричными исчезающими циклами.
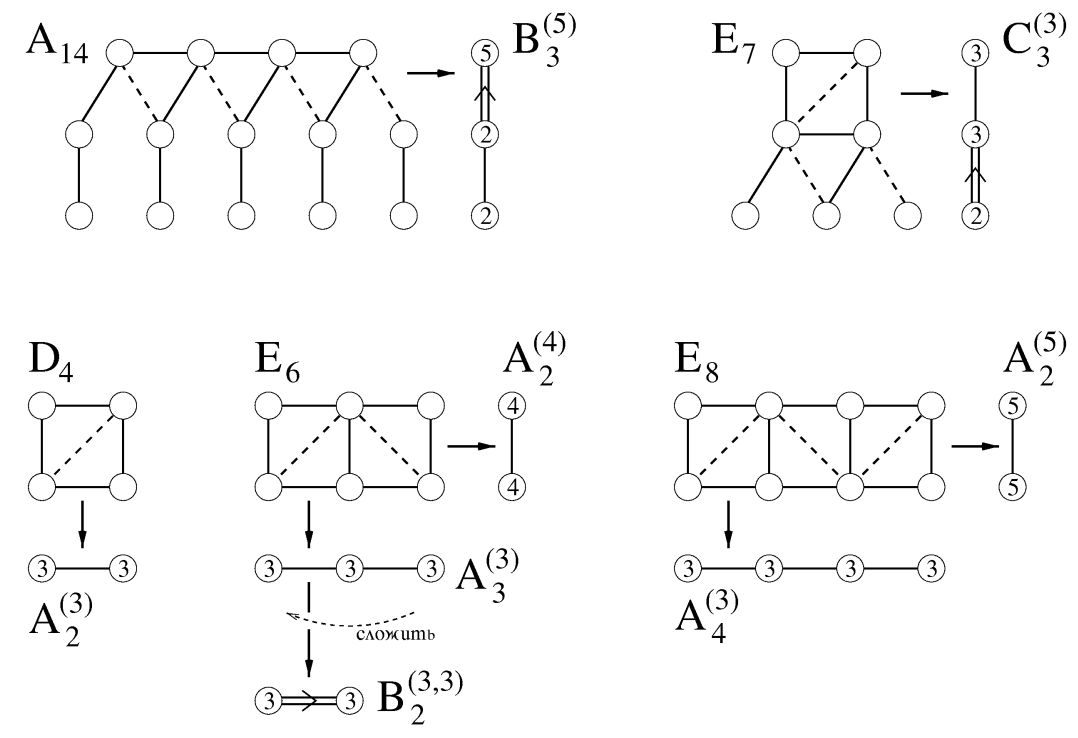

Рис. 8. Стягивание диаграмм групп Вейля на диаграммы унитарных групп отражений

Все это указьвает на способ получения диаграмм унитарных групп (рис. 3 ) из подходящих диаграмм объемлющих групп Вейля (указанных в последнем столбце таблицы теоремы 5.2) путем стягивания поддиаграмм $A_{m-1}$ в "короткие" вершины и склеи- 
вания подмножеств из $m$ несвязанных вершин в “длинные" вершины. Дополнительно к этому необходимо также проверить, вьполняются ли требуемые соотношения между образуюшими (или же между эрмитовыми произведениями получаемых корней), т.е. правильно ли операция стягивания-складьвания действует на ребрах.

Результаты применения описанного алгоритма приведены на рис. 8. Мы предполагаем, что размерность слоя Милнора кратна четырем. Диаграммы абсолютных особенностей далеки от стандартных диаграмм Дынкина групп Вейля. Как обычно, все их вершины имеют квадрат 2 , а сплошное ребро означает, что скалярное произведение корней равно -1. Пунктирное ребро отвечает произведению 1.

\section{СПИСОК ЛИТЕРАТУРЫ}

[1] Арнольд В.И. Нормальные формы функций вблизи вырожденных критических точек, группы Вейля $A_{k}, D_{k}, E_{k}$ и лагранжевы особенности // Функц. анализ и его прил. 1972. T. 6. № 4. C. $3-25$.

[2] Арнольд В.И. Критические точки функций на многообразии с краем, простые группы Ли $B_{k}, C_{k}, F_{4}$ и особенности эволют // УМН. 1978. Т. 33. № 5. С. 91-105.

[3] А рнольд В.И., Варченко А. Н., Гусейн-Заде С. М. Особенности дифференцируемых отображений. І. Классификация критических точек, каустик и волновых фронтов. М.: Наука, 1982.

[4] Брискорн Э. Монодромия изолированных особенностей гиперповерхностей // Математика. 1972. Т. 15. № 4. C. 130-160.

[5] Brieskorn E. Die Fundamentalgruppe des Raumes der regulären Orbits einer endlichen komplexen Spiegelungsgruppe // Invent. Math. 1971. V. 12. P. 57-61.

[6] Broué M., Malle G. Zyklotomische Heckealgebren // Astérisque. 1993. V. 212. P. 119-189.

[7] Cohen A. M. Finite complex reflection groups // Ann. Sci. Ecole Norm. Sup. 1976. V. 9. P. $379-436$.

[8] Coxeter H.S. M. Finite groups generated by unitary reflections // Abh. Math. Sem. Univ. Hamburg. 1967. V. 31. P. 125-135.

[9] Coxeter H.S. M. Regular Complex Polytopes. London: Cambridge Univ. Press, 1974.

[10] Ebeling W., Gusein-Zade S. M. Suspensions of fat points and their intersection forms // Progr. Math. 1998. V. 162. P. 141-165.

[11] Габриэлов А. М. Матрищы пересечений для некоторых особенностей // Функц. анализ и его прил. 1973. Т. 7. №3. С. 8-32.

[12] Гивенталь А. Б. Скрученные формулы Пикара-Леффшеца // Функц. анализ и его прил. 1988. T. 22. № 1. C. 12-22.

[13] Гивенталь А. Б. Особые лагранжевы многообразия и их лагранжевы отображения // Современные проблемы математики. Новейшие достижения. Т. 33. М.: ВИНИТИ, 1988. C. $55-112$.

[14] Ляшко О. В. Классификация критических точек функций на многообразии с особым краем // Функц. анализ и его прил. 1983. Т. 17. №3. С. 28-36.

[15] Милнор Дж. Особые точки комплексных гиперповерхностей. М.: Мир, 1971.

[16] Orlik P., Solomon L. Discriminants in the invariant theory of reflection groups // Nagoya Math. J. 1988. V. 109. P. 23-45.

[17] Popov V. L. Discrete complex reflection groups // Comm. Math. Inst., Rijksuniv. Utr. 1982. V. 15. P. 1-89.

[18] Шербак О.П. Волновые фронты и группы отражений // УМН. 1988. Т. 43. №3. C. $125-160$

[19] Shephard G. C., Todd J. A. Finite unitary reflection groups // Canad. J. Math. 1954. V. 6. P. 274-304.

[20] Siersma D. Singularities of functions on boundaries, corners, etc. // Quart. J. Math. 1981. V. 32. P. 119-127. 
[21] Tibar M. On isolated cyclic quotients with simplest complex link // Abh. Math. Sem. Univ. Hamburg. 1995. V. 65. P. 205-214.

[22] Wassermann G. Classification of singularities with compact abelian symmetry // Regensburger Math. Schr. 1977. V. 1. P. 1-284.

The University of Liverpool

Поступила в редакцию

E-mail: goryunov@liv.ac.uk

29.04.1999 